\title{
ON THE LINEAR INDEPENDENCE OF CERTAIN COHOMOLOGY CLASSES IN THE CLASSIFYING SPACE FOR SUBFOLIATIONS
}

\author{
DEMETRIO DOMÍNGUEZ
}

\begin{abstract}
The purpose of this paper is to establish the linear independence of certain cohomology classes in the Haefliger classifying space $B \Gamma_{\left(q_{1}, q_{2}\right)}$ for subfoliations of codimension $\left(q_{1}, q_{2}\right)$. The classes considered are of secondary type, not belonging to the subalgebra of $H\left(B \Gamma_{\left(q_{1}, q_{2}\right)}, R\right)$ generated by the union of the universal characteristic classes for foliations of codimension $q_{1}$ and $q_{2}$ respectively, and are elements of the kernel of the canonical homomorphism $H\left(B \Gamma_{\left(q_{1}, q_{2}\right)}, R\right) \rightarrow H\left(B \Gamma_{q_{1}} \times B \Gamma_{d}, R\right)$ with $d=q_{2}-q_{1}>0$.
\end{abstract}

\section{INTRODUCTION}

Let $M$ be an $n$-dimensional manifold and $T M$ its tangent bundle. A $\left(q_{1}, q_{2}\right)$-codimensional subfoliation on $M$ is a couple $\left(F_{1}, F_{2}\right)$ of integrable subbundles $F_{i}$ of $T M$ of dimension $n-q_{i}, i=1,2$, with $F_{2}$ being at the same time a subbundle of $F_{1}$. Therefore, for each $i=1,2, F_{i}$ defines a $q_{i}$ codimensional foliation on $M, d=q_{2}-q_{1} \geq 0$, and the leaves of $F_{1}$ contain those of $F_{2}$, Moussu [18], Feigin [9], and Cordero-Masa [5] have studied the (exotic) characteristic homomorphism of a subfoliation $\left(F_{1}, F_{2}\right)$, and Carballés [3] has generalized Cordero-Masa's construction, introducing the characteristic homomorphism $\Delta_{*}(P): H\left(W(g, H)_{I}\right) \rightarrow H_{\mathrm{DR}}(M)$ of an $\left(F_{1}, F_{2}\right)$-foliated principal bundle $P=P_{1}+P_{2}$ over $M$ of structure group $G=G_{1} \times G_{2}$. The author has computed in [6] the cohomology algebras $H\left(W(g, H)_{I}\right)$ and has given some geometric interpretations for the Godbillon-Vey classes of a subfoliation. Finally, the author has evaluated in [7] the characteristic homomorphism of a subfoliation for the particular case of locally homogeneous subfoliations, using the techniques of Kamber-Tondeur [16] and Carballés [3], and has given several examples of such subfoliations with nontrivial secondary or exotic characteristic classes which do not belong to the subalgebra of $H_{\mathrm{DR}}(M)$ generated by the characteristic classes of the two foliations.

In this paper, using some of the results obtained in $[6,7]$, we prove the linear independence of certain cohomology classes in the Haefliger classifying space $B \Gamma_{\left(q_{1}, q_{2}\right)}$ for subfoliations of codimension $\left(q_{1}, q_{2}\right)=(d+1,2 d+1)$ with $d \geq$ 1 . These classes are universal secondary characteristic classes for subfoliations of codimension $\left(q_{1}, q_{2}\right)$, not belonging to the subalgebra of $H\left(B \Gamma_{\left(q_{1}, q_{2}\right)}, R\right)$ generated by the union of the universal characteristic classes for foliations of

Received by the editors January 26, 1989 and, in revised form, November 2, 1989.

1980 Mathematics Subject Classification (1985 Revision). Primary 53C12, 57R32, 55R40. 
codimension $q_{1}$ and $q_{2}$ respectively. Furthermore, the classes considered are in the kernel of the canonical homomorphism

$$
H\left(B \Gamma_{\left(q_{1}, q_{2}\right)}, R\right) \rightarrow H\left(B \Gamma_{q_{1}} \times B \Gamma_{d}, R\right),
$$

where $B \Gamma_{q_{1}}$ (resp. $B \Gamma_{d}$ ) is the Haefliger classifying space for foliations of codimension $q_{1}$ (resp. $d$ ). A similar result holds for cohomology classes in the Haefliger classifying space $B \Gamma_{\left(q_{1}, q_{2}\right)}^{+}$(resp. $\left.F \Gamma_{\left(q_{1}, q_{2}\right)}\right)$ for subfoliations of codimension $\left(q_{1}, q_{2}\right)$ with oriented normal bundle (resp. with trivialized normal bundle).

The paper is structured as follows. In $\S 2$ we define the classifying space $B \Gamma_{\left(q_{1}, q_{2}\right)}$ for subfoliations of codimension $\left(q_{1}, q_{2}\right)$. In $\S 3$ we introduce the universal characteristic homomorphism $\Delta_{*}: H\left(W \mathrm{O}_{I}\right) \rightarrow H\left(B \Gamma_{\left(q_{1}, q_{2}\right)}, R\right)$ for subfoliations of codimension $\left(q_{1}, \dot{q}_{2}\right)$ using the techniques of Bott [2] and Cordero-Masa [5]. $\S 4$ is devoted to the computation of the canonical homomorphism $p_{*}: H\left(W \mathrm{O}_{I}\right) \rightarrow H\left(W \mathrm{O}_{q_{1}}\right) \otimes H\left(W \mathrm{O}_{d}\right)$ using the author's techniques [6]. Finally, in $\S 5$ the results obtained in $\S \S 3,4$ and [7] are used in order to establish the results of the preceding paragraph.

Throughout this paper all manifolds, foliations, and subfoliations are of type $C^{\infty}$, and cohomology groups are taken with real coefficients. We also adopt the notations of $[3,6,7,16]$.

\section{THE CLASSIFYING SPACE FOR SUBFOLIATIONS}

In this section we define a classifying space for subfoliations. For this purpose, the techniques used by Haefliger in [12] will be adopted here.

Let $\left(q_{1}, q_{2}\right)$ be a couple of integers $q_{i}$ with $0 \leq q_{1} \leq q_{2}$. Consider the subgroupoid $\Gamma=\Gamma_{\left(q_{1}, q_{2}\right)} \subset \Gamma_{q_{2}}$ of germs of local diffeomorphisms of $R^{q_{2}}=$ $R^{q_{1}} \times R^{d}$ preserving the foliation on $R^{q_{2}}$ defined by the canonical projection into $R^{q_{1}}$, where $d=q_{2}-q_{1} \geq 0$. Let $G=\operatorname{GL}\left(q_{1}, q_{2}\right) \subset \operatorname{GL}\left(q_{2}\right)$ be the subgroup of matrices of the form

$$
\left(\begin{array}{l|l}
A & 0 \\
\hline * & B
\end{array}\right)
$$

with $A \in \mathrm{GL}\left(q_{1}\right)$ and $B \in \mathrm{GL}(d)$. Clearly, $\mathrm{GL}\left(q_{1}\right) \times \mathrm{GL}(d) \subset G$ is a deformation retract. The differential defines a continuous homomorphism $\Gamma \rightarrow G$, and it induces a continuous map $\nu: B \Gamma \rightarrow B G$ classifying the normal bundle of the universal $\Gamma$-structure on the Haefliger classifying space $B \Gamma$ for $\Gamma$.

Now, let $\left(F_{1}, F_{2}\right)$ be a $\left(q_{1}, q_{2}\right)$-codimensional subfoliation on an $n$-dimensional manifold $M$. Then $\left(F_{1}, F_{2}\right)$ is given by an open cover of $M$ by coordinate charts $\left\{U_{\alpha}\right\}_{\alpha \in \Lambda}$ with local coordinate functions $x_{1}^{\alpha}, \ldots, x_{n}^{\alpha}$ satisfying

$$
\frac{\partial x_{i}^{\beta}}{\partial x_{a}^{\alpha}}=\frac{\partial x_{i}^{\beta}}{\partial x_{u}^{\alpha}}=\frac{\partial x_{a}^{\beta}}{\partial x_{u}^{\alpha}}=0 \text { on } U_{\alpha} \cap U_{\beta} \text { for } 1 \leq i \leq q_{1}<a \leq q_{2}<u \leq n .
$$

It follows that there is an open cover $\left\{U_{\alpha}\right\}_{\alpha \in \Lambda}$ of $M$ and submersions $f_{\alpha}: U_{\alpha} \rightarrow$ $R^{q_{2}}=R^{q_{1}} \times R^{d}$ such that

$$
\left(\left.F_{1}\right|_{U_{\alpha}},\left.F_{2}\right|_{U_{\alpha}}\right)=\left(f_{\alpha}^{-1}\left(0 \times T R^{d}\right), f_{\alpha}^{-1}(0)\right) \text { for } \alpha \in \Lambda .
$$

Hence, using the techniques of Haefliger [12], we obtain the following result. 
Proposition 2.1. $A\left(q_{1}, q_{2}\right)$-codimensional subfoliation $\left(F_{1}, F_{2}\right)$ on $M$ is classified (up to homotopy) by a continuous map $f: M \rightarrow B \Gamma$, and its normal bundle $\nu\left(F_{1}, F_{2}\right)=\nu F_{1} \oplus\left(F_{1} / F_{2}\right)$ is classified by the composition

$$
\begin{aligned}
M \stackrel{f}{\rightarrow} & B \Gamma \stackrel{\nu}{\rightarrow} B G \approx B\left(\mathrm{GL}\left(q_{1}\right) \times \mathrm{GL}(d)\right) \\
& \approx B \mathrm{GL}\left(q_{1}\right) \times B \mathrm{GL}(d) \approx B \mathrm{O}\left(q_{1}\right) \times B \mathrm{O}(d) .
\end{aligned}
$$

Remarks. (1) It is clear that $F_{i}$ is classified by the map $f_{i}=\phi_{i} \circ f: M \rightarrow$ $B \Gamma \rightarrow B \Gamma_{q_{i}}, i=1,2$, where $\phi_{i}$ is the canonical map. Of course, the map $\nu \circ f: M \rightarrow B \Gamma \rightarrow B G$ classifies the vector bundle $\nu F_{2} \cong \nu\left(F_{1}, F_{2}\right)$ with structure group $G=\mathrm{GL}\left(q_{1}, q_{2}\right)$.

(2) Similarly, a double foliation on $M$ given by two transverse foliations $F_{1}$ and $F_{0}$ of codimension $q_{1}$ and $d$ respectively is classified (up to homotopy) by a continuous map $f: M \rightarrow B\left(\Gamma_{q_{1}} \times \Gamma_{d}\right) \approx B \Gamma_{q_{1}} \times B \Gamma_{d}$. The $\left(q_{1}, q_{2}\right)$ codimensional subfoliation $\left(F_{1}, F_{2}\right)=\left(F_{1}, F_{1} \cap F_{0}\right)$ on $M$ is classified by the map $\phi \circ f: M \rightarrow B\left(\Gamma_{q_{1}} \times \Gamma_{d}\right) \rightarrow B \Gamma$, and $F_{1}$ (resp. $\left.F_{0}\right)$ is classified by the map $\tilde{\phi}_{1} \circ f: M \rightarrow B\left(\Gamma_{q_{1}} \times \Gamma_{d}\right) \rightarrow B \Gamma_{q_{1}}$ (resp. by the map $\tilde{\phi}_{0} \circ f: M \rightarrow B\left(\Gamma_{q_{1}} \times \Gamma_{d}\right) \rightarrow$ $B \Gamma_{d}$ ), where $\phi, \tilde{\phi}_{1}$, and $\tilde{\phi}_{0}$ denote the canonical maps.

(3) In the same way, a $\left(q_{1}, q_{2}\right)$-codimensional subfoliation $\left(F_{1}, F_{2}\right)$ with trivialized normal bundle on $M$ (in the sense of Cordero-Masa [5]) is classified (up to homotopy) by a continuous map $f: M \rightarrow F \Gamma$, where $F \Gamma$ denotes the homotopy theoretic fiber of the map $\nu: B \Gamma \rightarrow B G$. Analogously, a $\left(q_{1}, q_{2}\right)$ codimensional subfoliation $\left(F_{1}, F_{2}\right)$ with oriented normal bundle on $M$ (in the sense of [6]) is classified (up to homotopy) by a continuous map $f: M \rightarrow$ $B \Gamma^{+}$, where $\Gamma^{+}=\Gamma_{\left(q_{1}, q_{2}\right)}^{+} \subset \Gamma$ is the subgroupoid of all $\gamma \in \Gamma$ such that the differential of $\gamma$ belongs to the connected component $G_{0}$ of the group $G$.

\section{Characteristic Classes of Subfoliations}

In this section the universal characteristic classes for subfoliations are discussed.

Let

$$
\nu^{*}: H\left(B \mathrm{O}\left(q_{1}\right), R\right) \otimes H(B \mathrm{O}(d), R) \cong H(B G, R) \rightarrow H(B \Gamma, R)
$$

be the homomorphism induced by the map $B \Gamma \stackrel{\nu}{\rightarrow} B G \approx B \mathrm{O}\left(q_{1}\right) \times B \mathrm{O}(d)$ in cohomology. Then, using the techniques of Bott [2], from Theorem 3.9 in [5] we obtain Bott's obstruction theorem for subfoliations (actually, for $\Gamma$-structures):

Theorem 3.1. $\nu^{*}\left(H^{i}\left(B \mathrm{O}\left(q_{1}\right), R\right) \otimes H^{j}(B \mathrm{O}(d), R)\right)=0$ if at least one of the inequalities $i>2 q_{1}, i+j>2 q_{2}$ is satisfied.

Similarly, since the characteristic homomorphism for subfoliations is natural with respect to subfoliation preserving maps, we have the following

Theorem 3.2. There exists a unique homomorphism

$$
\Delta_{*}: H\left(W \mathrm{O}_{I}\right) \cong H\left(W\left(\operatorname{gl}\left(q_{1}\right) \oplus \operatorname{gl}(d), \mathrm{O}\left(q_{1}\right) \times \mathrm{O}(d)\right)_{I}\right) \rightarrow H(B \Gamma, R)
$$

such that $\Delta_{*\left(F_{1}, F_{2}\right)}=f^{*} \circ \Delta_{*}: H\left(W \mathrm{O}_{I}\right) \rightarrow H(B \Gamma, R) \rightarrow H(M, R) \cong H_{\mathrm{DR}}(M)$ for any $\left(q_{1}, q_{2}\right)$-codimensional subfoliation $\left(F_{1}, F_{2}\right)$ on a manifold $M$ with classifying map $f: M \rightarrow B \Gamma$, where $\Delta_{*\left(F_{1}, F_{2}\right)}$ denotes the characteristic homomorphism of $\left(F_{1}, F_{2}\right)$ as defined in [5]. 
Definition 3.3. $\Delta_{*}$ is called the universal characteristic homomorphism for subfoliations of codimension $\left(q_{1}, q_{2}\right)$ and the elements of $\operatorname{Im} \Delta_{*} \subset H(B \Gamma, R)$ are called the universal characteristic classes for subfoliations of codimension $\left(q_{1}, q_{2}\right)$.

Our purpose is to obtain information about $\operatorname{Im} \Delta_{*} \subset H(B \Gamma, R)$. In the computation of the homomorphism $\Delta_{*}$, the following result is interesting.

Proposition 3.4. The diagram

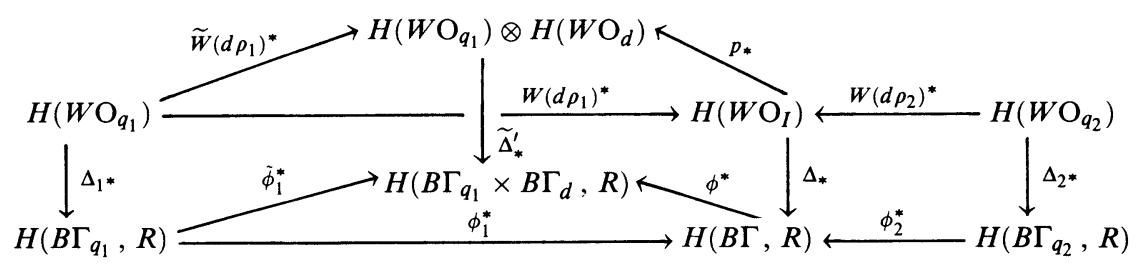

is commutative, where the vertical maps are the universal characteristic homomorphisms (with $\widetilde{\Delta}_{*}^{\prime}$ the universal characteristic homomorphism for double foliations of codimension $q_{1}$ and $d$ respectively), and the horizontal maps are the canonical homomorphisms.

Proof. This follows from Theorem 5.2 in [5] and Theorem 4.6 in [6].

Remarks. (1) It is clear that the canonical homomorphisms $\phi_{1}^{*}, \tilde{\phi}_{1}^{*}, W\left(d \rho_{1}\right)^{*}$, and $\widetilde{W}\left(d \rho_{1}\right)^{*}$ are injective, and that $\operatorname{Im} \phi_{i}^{*} \Delta_{i *} \subset \operatorname{Im} \Delta_{*}, i=1,2$.

(2) For $q_{1}=q_{2}=q$, and for $q_{1}=0$ and $q_{2}=q$, the results obtained above are reduced to the ordinary case of foliations of codimension $q$. On the other hand, $B \Gamma$ can also be considered as the classifying space for foliated manifolds $(M, F)$ with $F$ of codimension $q_{1}$ and $M$ of dimension $q_{2}$.

(3) The universal characteristic homomorphism

$$
\widetilde{\Delta}_{*}: H\left(W_{I}\right) \cong H\left(W\left(\operatorname{gl}\left(q_{1}\right) \oplus \operatorname{gl}(d)\right)_{I}\right) \rightarrow H(F \Gamma, R)
$$

(resp. $\left.\quad \Delta_{*}^{\prime}: H\left(W\left(\operatorname{gl}\left(q_{1}\right) \oplus \operatorname{gl}(d), \mathrm{SO}\left(q_{1}\right) \times \mathrm{SO}(d)\right)_{I}\right) \rightarrow H\left(B \Gamma^{+}, R\right)\right)$ for subfoliations of codimension $\left(q_{1}, q_{2}\right)$ with trivialized normal bundle (resp. with oriented normal bundle) is constructed in an analogous way and results similar to those announced in Proposition 3.4 are obtained. Moreover, there is a commutative diagram

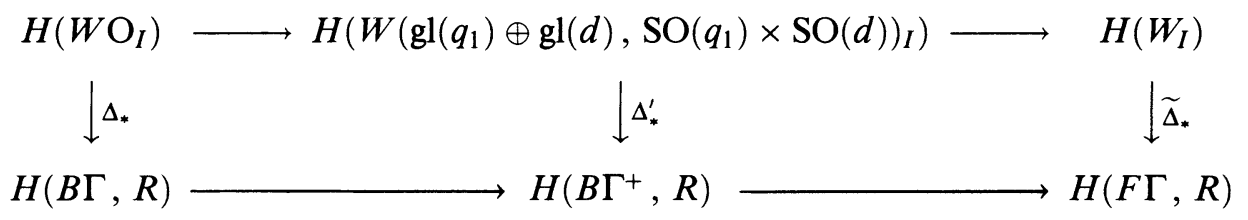

with canonical horizontal maps.

\section{THE COMPUTATION OF THE HOMOMORPHISM $p_{*}$}

In this section, using the notations of $\S 3$ in [6], we give the computation of the canonical homomorphism $p_{*}: H\left(W \mathrm{O}_{I}\right) \rightarrow H\left(W \mathrm{O}_{q_{1}}\right) \otimes H\left(W \mathrm{O}_{d}\right)$ with $0<q_{1}<q_{2}$ and $d=q_{2}-q_{1}>0$. 
Proposition 4.1. The cohomology classes in $H\left(W \mathrm{O}_{I}\right)$ of the cocycles

$$
\begin{aligned}
z_{\left(i, i^{\prime}, j, j^{\prime}\right)}= & y_{(i)} \wedge y_{\left(i^{\prime}\right)}^{\prime} \otimes c_{(j)} c_{\left(j^{\prime}\right)}^{\prime} \\
= & y_{i_{1}} \wedge \cdots \wedge y_{i_{s}} \wedge y_{i_{1}^{\prime}}^{\prime} \wedge \cdots \wedge y_{i_{s^{\prime}}^{\prime}}^{\prime} \\
& \otimes c_{1}^{j_{1}} \cdots c_{q_{1}}^{j_{q_{1}}} c_{1}^{\prime j_{1}^{\prime}} \cdots c_{d}^{\prime j_{d}^{\prime}} \in W \mathrm{O}_{I}
\end{aligned}
$$

of the Vey basis (with $2 p_{1}=\operatorname{deg} c_{(j)}, 2 p_{2}=\operatorname{deg} c_{\left(j^{\prime}\right)}^{\prime}$, and $p=p_{1}+p_{2}$ ) satisfying the conditions:

(1) $0 \leq p_{1} \leq q_{1}, 0 \leq p_{2} \leq d, 0 \leq s \leq\left[\left(q_{1}+1\right) / 2\right]$, and $0 \leq s^{\prime} \leq[(d+1) / 2]$;

(2) $i_{0}+p_{1} \geq q_{1}+1$ and $i_{0}^{\prime}+p \geq q_{2}+1$;

(3) $i_{0} \leq j_{0}$ and $i_{0}^{\prime} \leq j_{0}^{\prime}$

are mapped under the homomorphism $p_{*}: H\left(W \mathrm{O}_{I}\right) \rightarrow H\left(W \mathrm{O}_{q_{1}}\right) \otimes H\left(W \mathrm{O}_{d}\right)$ onto a basis of $\operatorname{Im} p_{*} \subset H\left(W \mathrm{O}_{q_{1}}\right) \otimes H\left(W \mathrm{O}_{d}\right)$.

Proof. Let $C$ be the set of elements $z_{\left(i, i^{\prime}, j, j^{\prime}\right)}$ such that $0 \leq p_{1} \leq q_{1}, 0 \leq$ $p \leq q_{2}, 0 \leq s \leq\left[\left(q_{1}+1\right) / 2\right]$, and $0 \leq s^{\prime} \leq[(d+1) / 2]$. Let $C_{\mathrm{a}}, C_{\mathrm{b}}$, and $C_{\mathrm{c}}$ be the sets of elements $z_{\left(i, i^{\prime}, j, j^{\prime}\right)} \in C$ satisfying the conditions:

( $\left.C_{\mathrm{a}}\right) i_{0}>i_{0}^{\prime}, i_{0}^{\prime}<j_{0}, i_{0}^{\prime} \leq j_{0}^{\prime}$, and $i_{0}^{\prime}+p \geq q_{2}+1$;

$\left(C_{\mathrm{b}}\right) i_{0} \leq i_{0}^{\prime}, i_{0} \leq j_{0}, i_{0} \leq j_{0}^{\prime}, i_{0}+p_{1}<q_{1}+1$, and $i_{0}+p \geq q_{2}+1$;

$\left(C_{\mathrm{c}}\right) i_{0} \leq i_{0}^{\prime}, i_{0} \leq j_{0}, i_{0}^{\prime} \leq j_{0}^{\prime}, i_{0}+p_{1} \geq q_{1}+1$, and $i_{0}^{\prime}+p \geq q_{2}+1$

respectively. In particular, we have $C_{\mathrm{a}} \cap C_{\mathrm{b}}=C_{\mathrm{a}} \cap C_{\mathrm{c}}=C_{\mathrm{b}} \cap C_{\mathrm{c}}=\varnothing$. It is clear that the elements $z_{\left(i, i^{\prime}, j, j^{\prime}\right)} \in C_{\mathrm{a}} \cup C_{\mathrm{b}} \cup C_{\mathrm{c}}$ are cocycles and that the Vey basis of $H\left(W \mathrm{O}_{I}\right)$ is given by the cohomology classes of these cocycles.

Clearly, we have $p_{*}\left[z_{\left(i, i^{\prime}, j, j^{\prime}\right)}\right]=0$ for $z_{\left(i, i^{\prime}, j, j^{\prime}\right)} \in C_{\mathrm{a}} \cup C_{\mathrm{b}} \cup C_{\mathrm{c}}$ such that $d<p_{2} \leq q_{2}$. It follows that $p_{*}\left[z_{\left(i, i^{\prime}, j, j^{\prime}\right)}\right]=0$ for $z_{\left(i, i^{\prime}, j, j^{\prime}\right)} \in C_{\mathrm{b}}$. On the other hand, consider the subspace $\widetilde{C}_{\mathrm{a}}$ (resp. $\left.\widetilde{C}_{\mathrm{c}}\right)$ of $H\left(W \mathrm{O}_{I}\right)$ spanned by the classes of the cocycles $z_{\left(i, i^{\prime}, j, j^{\prime}\right)} \in C_{\mathrm{a}}$ such that $0 \leq p_{2} \leq d$ and $i_{0} \leq j_{0}$ (resp. of the cocycles $z_{\left(i, i^{\prime}, j, j^{\prime}\right)} \in C_{\mathrm{c}}$ such that $\left.0 \leq p_{2} \leq d\right)$. Then it is easy to see that the $R$-linear map

$$
\left.p_{*}\right|_{\widetilde{C}}: \widetilde{C} \rightarrow H\left(W \mathrm{O}_{q_{1}}\right) \otimes H\left(W \mathrm{O}_{d}\right)
$$

is injective, where $\widetilde{C}=\widetilde{C}_{\mathrm{a}} \oplus \widetilde{C}_{\mathrm{c}}$.

Finally, for $z_{\left(i, i^{\prime}, j, j^{\prime}\right)} \in C_{\mathrm{a}}$ with $0 \leq p_{2} \leq d$ and $j_{0}<i_{0}$, we obtain

$$
\begin{aligned}
p_{*}\left[z_{\left(i, i^{\prime}, j, j^{\prime}\right)}\right] & =\left[y_{(i)} \otimes c_{(j)}\right] \otimes\left[y_{\left(i^{\prime}\right)}^{\prime} \otimes c_{\left(j^{\prime}\right)}^{\prime}\right] \\
& =\sum_{t=1}^{s}(-1)^{t+1} p_{*}\left[y_{j_{0}} \wedge y_{i_{1}} \wedge \cdots \wedge \hat{y}_{i_{t}} \wedge \cdots \wedge y_{i_{s}} \wedge y_{\left(i^{\prime}\right)}^{\prime} \otimes c_{i_{t}} \cdot \Phi \cdot c_{\left(j^{\prime}\right)}^{\prime}\right],
\end{aligned}
$$

where $\Phi=c_{1}^{j_{1}} \cdots c_{j_{0}}^{j_{j_{0}}-1} \cdots c_{q_{1}}^{j_{q_{1}}}$ (with $p_{*}\left[z_{\left(i, i^{\prime}, j, j^{\prime}\right)}\right]=0$ for $s=0$ ); it follows that $p_{*}\left[z_{\left(i, i^{\prime}, j, j^{\prime}\right)}\right] \in p_{*} \widetilde{C}_{\mathrm{a}}$. Whence $\operatorname{Im} p_{*}=p_{*} \widetilde{C}$.

In a similar way, we obtain the following

Proposition 4.2. An $R$-basis of $\operatorname{Ker} p_{*} \subset H\left(W \mathrm{O}_{I}\right)$ is given by the union of the classes $\left[z_{\left(i, i^{\prime}, j, j^{\prime}\right)}\right]$ of the Vey basis of $H\left(W \mathrm{O}_{I}\right)$ such that $d<p_{2} \leq q_{2}$ and the classes of the cocycles

$$
\begin{gathered}
z_{\left(i, i^{\prime}, j, j^{\prime}\right)}^{\prime}=z_{\left(i, i^{\prime}, j, j^{\prime}\right)}-\sum_{t=1}^{s}(-1)^{t+1} y_{j_{0}} \wedge y_{i_{1}} \wedge \cdots \wedge \hat{y}_{i_{t}} \\
\wedge \cdots \wedge y_{i_{s}} \wedge y_{\left(i^{\prime}\right)}^{\prime} \otimes c_{i_{t}} \cdot \Phi \cdot c_{\left(j^{\prime}\right)}^{\prime}
\end{gathered}
$$


such that $0 \leq p_{1} \leq q_{1}, 0 \leq p_{2} \leq d, 0 \leq s \leq\left[\left(q_{1}+1\right) / 2\right], 0 \leq s^{\prime} \leq[(d+1) / 2]$, $i_{0}>j_{0}>i_{0}^{\prime}, i_{0}^{\prime} \leq j_{0}^{\prime}$, and $i_{0}^{\prime}+p \geq q_{2}+1$, where $\Phi=c_{1}^{j_{1}} \cdots c_{j_{0}}^{j_{j_{0}}-1} \cdots c_{q_{1}}^{j_{q_{1}}}$ (with $z_{\left(i, i^{\prime}, j, j^{\prime}\right)}^{\prime}=z_{\left(i, i^{\prime}, j, j^{\prime}\right)}$ for $\left.s=0\right)$.

Let $\widetilde{C} \subset H\left(W \mathrm{O}_{I}\right)$ be the subspace spanned by the cohomology classes of the cocycles $z_{\left(i, i^{\prime}, j, j^{\prime}\right)}$ considered in Proposition 4.1. Let $\widetilde{C}^{\prime} \subset \widetilde{C}$ be a subspace with $\widetilde{C}^{\prime} \neq 0$, such that $\left(p_{*} \mid \widetilde{C}\right)^{-1}\left(\operatorname{Ker} \widetilde{\Delta}_{*}^{\prime}\right) \cap \widetilde{C}^{\prime}=0$ (evidently, the results of Kamber-Tondeur [17] imply that there is a subspace $\widetilde{C}^{\prime} \subset \widetilde{C}$ of dimension $2^{\left[\left(q_{1}+1\right) / 2\right]-1}\left(2^{[(d+1) / 2]-1}+1\right)$ satisfying the property above), where $\tilde{\Delta}_{*}^{\prime}=\mu \circ\left(\Delta_{1 *} \otimes \Delta_{0 *}\right): H\left(W \mathrm{O}_{q_{1}}\right) \otimes H\left(W \mathrm{O}_{d}\right) \rightarrow H\left(B \Gamma_{q_{1}} \times B \Gamma_{d}, R\right)$ denotes the universal characteristic homomorphism for double foliations of codimension $q_{1}$ and $d$ respectively, $\Delta_{1 *}$ (resp. $\Delta_{0 *}$ ) being the universal characteristic homomorphism for foliations of codimension $q_{1}$ (resp. $d$ ), and $\mu$ the cohomology cross product (clearly, the homomorphism $\mu$ is injective). Then, from Propositions 3.4, 4.1, and 4.2 we obtain the following result.

Corollary 4.3. (i) The R-linear maps

$$
\left.\Delta_{*}\right|_{\widetilde{C}^{\prime}}: \widetilde{C}^{\prime} \rightarrow H(B \Gamma, R) \text { and }\left.\phi^{*}\right|_{\Delta_{*} \widetilde{C}^{\prime}}: \Delta_{*} \widetilde{C}^{\prime} \rightarrow H\left(B \Gamma_{q_{1}} \times B \Gamma_{d}, R\right)
$$

are injective.

(ii) We have

$$
\begin{gathered}
\Delta_{*} \operatorname{Ker} p_{*} \subset \operatorname{Ker} \phi^{*} \subset H(B \Gamma, R), \\
\operatorname{Im} \Delta_{*}=\Delta_{*} \widetilde{C}+\Delta_{*} \operatorname{Ker} p_{*} \subset \Delta_{*} \widetilde{C}+\operatorname{Ker} \phi^{*} \subset H(B \Gamma, R), \\
\Delta_{*} \widetilde{C} \cap \operatorname{Ker} \phi^{*}=\Delta_{*}\left(\left(p_{*} \mid \widetilde{C}\right)^{-1}\left(\operatorname{Ker} \widetilde{\Delta}_{*}^{\prime}\right)\right), \\
\Delta_{*}^{-1}\left(\operatorname{Ker} \phi^{*}\right)=\left(p_{*} \mid \widetilde{C}\right)^{-1}\left(\operatorname{Ker} \widetilde{\Delta}_{*}^{\prime}\right) \oplus \operatorname{Ker} p_{*} \subset \widetilde{C} \oplus \operatorname{Ker} p_{*}=H\left(W \mathrm{O}_{I}\right), \\
\operatorname{Im} \phi_{1}^{*} \Delta_{1 *} \subset \Delta_{*} \widetilde{C}, \quad \text { and } \operatorname{Im} \phi_{1}^{*} \Delta_{1 *} \cap \operatorname{Ker} \phi^{*}=0 .
\end{gathered}
$$

Corollary 4.4. Let $u$ be an element of $H\left(W \mathrm{O}_{I}\right)$. Then $u \in \Delta_{*}^{-1}\left(\operatorname{Ker} \phi^{*}\right)$ if and only if $\Delta_{*\left(F_{1}, F_{2}\right)} u=0 \in H_{\mathrm{DR}}(M)$ for any manifold $M$ and for any $\left(q_{1}, q_{2}\right)$ codimensional subfoliation $\left(F_{1}, F_{2}\right)$ on $M$ such that $\left(F_{1}, F_{2}\right)=\left(F_{1}, F_{1} \cap F_{0}\right)$ with $F_{0}$ a d-codimensional foliation on $M$.

Corollary 4.5. For the universal Godbillon-Vey classes, we have

(i) $\Delta_{*}\left[y_{1}^{\prime} \otimes c_{1}^{j} c_{1}^{\prime q_{2}-j}\right], \Delta_{*}\left[y_{1} \wedge y_{1}^{\prime} \otimes c_{1}^{j^{\prime}} c_{1}^{\prime q_{2}-j^{\prime}}\right] \in \Delta_{*} \operatorname{Ker} p_{*} \subset \operatorname{Ker} \phi^{*}$ for $0 \leq$ $j \leq q_{1}$ and $0 \leq j^{\prime} \leq q_{1}-1$. In particular, for the universal Godbillon-Vey class $\Delta_{2 *}\left[y_{1}^{\prime \prime} \otimes c_{1}^{\prime \prime q_{2}}\right] \in H^{2 q_{2}+1}\left(B \Gamma_{q_{2}}, R\right)$, we have

$$
\left(\phi_{2}^{*} \circ \Delta_{2 *}\right)\left[y_{1}^{\prime \prime} \otimes c_{1}^{\prime \prime q_{2}}\right]=\sum_{j=0}^{q_{1}}\left(\begin{array}{c}
q_{2}+1 \\
j
\end{array}\right) \Delta_{*}\left[y_{1}^{\prime} \otimes c_{1}^{j} c_{1}^{\prime q_{2}-j}\right] \in \Delta_{*} \operatorname{Ker} p_{*} .
$$

(ii) $\Delta_{*}\left[y_{1} \otimes c_{1}^{q_{1}}\right], \Delta_{*}\left[y_{1} \wedge y_{1}^{\prime} \otimes c_{1}^{q_{1}} c_{1}^{\prime d}\right] \in \Delta_{*} \widetilde{C}-\operatorname{Ker} \phi^{*}$.

Remark. In the same way, we can compute the canonical homomorphisms $\tilde{p}_{*}: H\left(W_{I}\right) \rightarrow H\left(W_{q_{1}}\right) \otimes H\left(W_{d}\right)$ and

$$
\begin{aligned}
p_{*}^{\prime}: & H\left(W\left(\mathrm{gl}\left(q_{1}\right) \oplus \operatorname{gl}(d), \mathrm{SO}\left(q_{1}\right) \times \mathrm{SO}(d)\right)_{I}\right) \\
& \rightarrow H\left(W\left(\operatorname{gl}\left(q_{1}\right), \mathrm{SO}\left(q_{1}\right)\right)_{q_{1}}\right) \otimes H\left(W(\mathrm{gl}(d), \mathrm{SO}(d))_{d}\right) .
\end{aligned}
$$


Results similar to those announced above are obtained. It is clear that the homomorphism $p_{*}^{\prime}$ is given by $\left.p_{*}^{\prime}\right|_{H\left(W \mathrm{O}_{I}\right)}=p_{*}, p_{*}^{\prime} e_{m}=e_{m}$, and $p_{*}^{\prime} e_{n}^{\prime}=e_{n}^{\prime}$, where $e_{m} \in I^{2 m}\left(\mathrm{SO}\left(q_{1}\right)\right)$ (resp. $\left.e_{n}^{\prime} \in I^{2 n}(\mathrm{SO}(d))\right)$ denotes the Pfaffian pclynomial for $q_{1}=2 m$ (resp. for $d=2 n$ ).

\section{EXAMPLES AND APPLICATIONS}

In this section, using the examples of locally homogeneous subfoliations (with nontrivial characteristic classes) given in [7], we show that $\Delta_{*} \operatorname{Ker} p_{*} \subset$ $H(B \Gamma, R)$ is nontrivial for $\left(q_{1}, q_{2}\right)=(d+1,2 d+1)$ with $d \geq 1$.

Let $H \subset G_{2} \subset G_{1} \subset \bar{G}$ be Lie groups, and $h \subset g_{2} \subset g_{1} \subset \bar{g}$ their Lie algebras. Assume that $H$ is closed in $\bar{G}$. Let $\bar{\Gamma} \subset \bar{G}$ be a discrete subgroup acting properly discontinuously and without fixed points on $\bar{G} / H$, so that $M=$ $\bar{\Gamma} \backslash \bar{G} / H$ is a manifold. A $\left(q_{1}, q_{2}\right)$-codimensional subfoliation $\left(F_{1}, F_{2}\right)$ on $M$ of the form $\left(F_{1}, F_{2}\right)=\left(F_{G_{1}}, F_{G_{2}}\right)$ is called a locally homogeneous subfoliation, where $F_{i}=F_{G_{i}}$ is the locally homogeneous foliation of codimension $q_{i}=$ $\operatorname{dim} \bar{g} / g_{i}$ on $M$, induced by the foliation on $\bar{G}$ defined by the right action of $G_{i}, i=1,2$. The computation of the characteristic homomorphism for locally homogeneous subfoliations has been described in [7].

Clearly, if $G_{1}$ and $G_{2}$ are connected or if $H$ is connected, then $\left(F_{1}, F_{2}\right)=$ $\left(F_{G_{1}}, F_{G_{2}}\right)$ is a subfoliation with oriented normal bundle. Similarly, for $H=$ $\{e\},\left(F_{1}, F_{2}\right)=\left(F_{G_{1}}, F_{G_{2}}\right)$ is a subfoliation with trivialized normal bundle.

Example 1. Let $\bar{G}=\mathrm{SL}(d+2), G_{1}=\operatorname{SL}(d+2,1)_{0}, G_{2}=\operatorname{SL}(d+2,2)_{0}$, $H=\mathrm{SO}(d)$, and $\bar{T} \subset \mathrm{SL}(d+2)$ be a discrete uniform and torsion-free subgroup (with $d \geq 1)$, where $\operatorname{SL}(d+2,1)_{0}$ (resp. $\left.\operatorname{SL}(d+2,2)_{0}\right)$ denotes the connected component of the group $\operatorname{SL}(d+2,1)$ (resp. of the group $\operatorname{SL}(d+2,2)$ ) of unimodular matrices of the form

$$
\left(\begin{array}{l|l}
\lambda & * \\
\hline 0 & A
\end{array}\right)
$$

with $A \in \mathrm{GL}(d+1)$ and $\lambda^{-1}=\operatorname{det} A$ (resp. of the form

$$
\left(\begin{array}{l|l|l}
\lambda_{1} & & * \\
\hline 0 & \frac{\lambda_{2}}{*} & * \\
\hline 0 & B
\end{array}\right)
$$

with $B \in \mathrm{GL}(d), \lambda_{1}, \lambda_{2} \in \mathrm{GL}(1)$, and $\left.\lambda_{1}^{-1}=\lambda_{2} \cdot \operatorname{det} B\right)$. Then, by virtue of Theorem 3.2 in [7], $M=\bar{\Gamma} \backslash \bar{G} / H$ is a connected compact orientable manifold and the canonical homomorphism $\gamma_{*}: H(\bar{g}, H) \rightarrow H_{\mathrm{DR}}(M)$ is injective. Furthermore, we have

$$
H(\bar{g}, H) \cong\left\{\begin{array}{l}
\bigwedge\left(\bar{y}_{3}, \bar{y}_{5}, \ldots, \bar{y}_{2 n-1}, \bar{y}_{d+1}, \bar{y}_{d+2}\right) \text { for } d=2 n-1, \\
\bigwedge\left(\bar{y}_{3}, \bar{y}_{5}, \ldots, \bar{y}_{2 n-1}, \bar{y}_{d+1}, \bar{y}_{d+2}\right) \otimes R\left[e_{n}^{\prime}\right] /\left(e_{n}^{\prime 2}\right) \text { for } d=2 n,
\end{array}\right.
$$

where the elements $\bar{y}_{i}$ are the relative suspensions of the Chern polynomials $\bar{c}_{i} \in I(\mathrm{SL}(d+2))=R\left[\bar{c}_{2}, \bar{c}_{3}, \ldots, \bar{c}_{d+2}\right]$ and $e_{n}^{\prime} \in I^{2 n}(\mathrm{SO}(2 n))$ is the Pfaffian polynomial.

On the other hand, consider the locally homogeneous subfoliation $\left(F_{1}, F_{2}\right)=$ $\left(F_{G_{1}}, F_{G_{2}}\right)$ of codimension $\left(q_{1}, q_{2}\right)=(d+1,2 d+1)$ with oriented normal bundle on $M$. Let

$$
\Delta_{*\left(F_{1}, F_{2}\right)}: H\left(W(\mathrm{gl}(d+1) \oplus \mathrm{gl}(d), \mathrm{SO}(d+1) \times \mathrm{SO}(d))_{I}\right) \rightarrow H_{\mathrm{DR}}(M)
$$


be the characteristic homomorphism of this subfoliation. Let $c_{(j)}=c_{1}^{j_{1}} \cdots c_{d+1}^{j_{d+1}}$ be a monomial of $\operatorname{deg} c_{(j)}=2(d+1-k)$ and $c_{\left(j^{\prime}\right)}^{\prime}=c_{1}^{\prime} j_{1}^{\prime} \cdots c_{d}^{\prime j_{d}^{\prime}}$ a monomial of $\operatorname{deg} c_{\left(j^{\prime}\right)}^{\prime}=2(d+k)$ with $0 \leq k \leq d+1$. Choose integers $t_{0}, t_{1}, \ldots, t_{n-1}$ such that $0 \leq t_{s} \leq s$ for $s=0,1, \ldots, n-1$, where $n=[(d+1) / 2]$. Now, consider in $H\left(W(\operatorname{gl}(d+1) \oplus \operatorname{gl}(d), \mathrm{SO}(d+1) \times \mathrm{SO}(d))_{I}\right)$ the classes of the cocycles

$$
z_{\left(i, j, j^{\prime}\right)}=y_{1} \wedge y_{2 i_{1}-1} \wedge \cdots \wedge y_{2 i_{t_{s}}-1} \wedge y_{1}^{\prime} \wedge y_{2 i_{t_{s}+1}-1}^{\prime} \wedge \cdots \wedge y_{2 i_{s}-1}^{\prime} \otimes c_{(j)} c_{\left(j^{\prime}\right)}^{\prime}
$$

for $2 \leq i_{1}<\cdots<i_{t_{s}}<i_{t_{s}+1}<\cdots<i_{s} \leq n, 0 \leq s \leq n-1$, where $z_{\left(i, j, j^{\prime}\right)}=$ $y_{1} \wedge y_{1}^{\prime} \otimes c_{(j)} c_{\left(j^{\prime}\right)}^{\prime}$ for $s=0, \quad z_{\left(i, j, j^{\prime}\right)}=y_{1} \wedge y_{2 i_{1}-1} \wedge \cdots \wedge y_{2 i_{s}-1} \wedge y_{1}^{\prime} \otimes c_{(j)} c_{\left(j^{\prime}\right)}^{\prime}$ for $s>0$ and $t_{s}=s$, and $z_{\left(i, j, j^{\prime}\right)}=y_{1} \wedge y_{1}^{\prime} \wedge y_{2 i_{1}-1}^{\prime} \wedge \cdots \wedge y_{2 i_{s}-1}^{\prime} \otimes c_{(j)} c_{\left(j^{\prime}\right)}^{\prime}$ for $s>0$ and $t_{s}=0$. It is clear that the classes $\left[z_{\left(i, j, j^{\prime}\right)} \otimes \Phi\right]$ belong to the kernel of the canonical homomorphism

$$
\begin{aligned}
p_{*}^{\prime}: & H\left(W\left(\operatorname{gl}\left(q_{1}\right) \oplus \operatorname{gl}(d), \mathrm{SO}\left(q_{1}\right) \times \mathrm{SO}(d)\right)_{I}\right) \\
& \rightarrow H\left(W\left(\operatorname{gl}\left(q_{1}\right), \mathrm{SO}\left(q_{1}\right)\right)_{q_{1}}\right) \otimes H\left(W(\operatorname{gl}(d), \mathrm{SO}(d))_{d}\right)
\end{aligned}
$$

for $1 \leq k \leq d+1$, where $\Phi=1$ if $d=2 n-1$, and $\Phi=1$ or $e_{n}^{\prime}$ if $d=2 n$. We have then the following result.

Theorem 5.1. For $k=0$ and $1<k \leq d+1$, we have the linearly independent secondary classes

$$
\Delta_{*\left(F_{1}, F_{2}\right)}\left[z_{\left(i, j, j^{\prime}\right)} \otimes \Phi\right]=\mu \cdot \gamma_{*}\left(\bar{y}_{2 i_{1}-1} \wedge \cdots \wedge \bar{y}_{2 i_{s}-1} \wedge \bar{y}_{d+1} \wedge \bar{y}_{d+2} \otimes \Phi\right)
$$

with $2 \leq i_{1}<\cdots<i_{t_{s}}<i_{t_{s}+1}<\cdots<i_{s} \leq n, 0 \leq s \leq n-1, \Phi=1$ if $d=2 n-1, \Phi=1$ or $e_{n}^{\prime}$ if $d=2 n$, and

$$
\mu=(-1)^{t_{s}}(d+2)(d+1)\left(a_{k k-1}-a_{k k}\right) \prod_{i=1}^{d+1}\left(\begin{array}{c}
d+2 \\
i
\end{array}\right)^{j_{i}} \cdot \prod_{i=1}^{d}\left(\begin{array}{c}
d+1 \\
i
\end{array}\right)^{j_{i}^{\prime}} \neq 0,
$$

where $a_{k k-1}, a_{k k} \in R$ (with $a_{k k-1}=0$ for $k=0$ ) are given by the polynomial

$$
\begin{aligned}
f_{k}(\lambda) & =\prod_{i=1}^{d}\left(\sum_{u=0}^{i}\left(\left(\begin{array}{c}
i \\
u
\end{array}\right) /\left(\begin{array}{c}
d+1 \\
u
\end{array}\right)\right) \lambda^{u}\right)^{j_{i}^{\prime}} \\
& =\sum_{v=0}^{d+k} a_{k v} \lambda^{v} \in R[\lambda], \quad a_{k v} \in R .
\end{aligned}
$$

The corresponding classes then span the subspace

$$
\begin{aligned}
& \gamma_{*}\left(\operatorname{Ideal}\left(\bar{y}_{d+2} \wedge \bar{y}_{d+1}\right)\right) \\
& \quad=\left\{\begin{array}{l}
\gamma_{*}\left(\left(\bar{y}_{d+2} \wedge \bar{y}_{d+1}\right) \cdot \wedge\left(\bar{y}_{3}, \bar{y}_{5}, \ldots, \bar{y}_{2 n-1}\right)\right) \text { for } d=2 n-1, \\
\gamma_{*}\left(\left(\bar{y}_{d+2} \wedge \bar{y}_{d+1}\right) \cdot \wedge\left(\bar{y}_{3}, \bar{y}_{5}, \ldots, \bar{y}_{2 n-1}\right) \otimes R\left[e_{n}^{\prime}\right] /\left(e_{n}^{\prime 2}\right)\right) \text { for } d=2 n
\end{array}\right.
\end{aligned}
$$

of $H_{\mathrm{DR}}(M)$ of dimension $2^{[d / 2]}$. For $k=1$, we have $\Delta_{*\left(F_{1}, F_{2}\right)}\left[z_{\left(i, j, j^{\prime}\right)} \otimes \Phi\right]=0$. Proof. It suffices to proceed as in the proof of Theorem 6.1 in [7]. It is easy to see that $a_{k k}=1$ for $k=0$. Thus we have only to show that $a_{k k-1}-a_{k k} \neq 0$ for $1<k \leq d+1$, and $a_{k k-1}-a_{k k}=0$ for $k=1$.

Now, using the $v$ th derivative of $f_{k}(\lambda), v=0,1, \ldots, d+k$, by a direct computation of $a_{k v}>0$ for $0 \leq v \leq d+k, 1 \leq k \leq d+1$, we then obtain

$$
a_{k v}<((v d+k) /(v d+v)) a_{k v-1} \text { for } 1<v \leq d+k, 1 \leq k \leq d+1 \text {. }
$$


It follows that $a_{k v}<a_{k v-1}$ for $\max (2, k) \leq v \leq d+k, 1 \leq k \leq d+1$. Hence

$$
a_{k k-1}-a_{k k}>0 \text { for } 1<k \leq d+1 \text {. }
$$

On the other hand, since $a_{k 0}=1$ and $a_{k 1}=(d+k) /(d+1)$ for $1 \leq k \leq$ $d+1$, we have $a_{k 0}-a_{k 1}=0$ for $k=1$. It follows that

$$
\Delta_{*}\left[z_{\left(i, j, j^{\prime}\right)} \otimes \Phi\right]=0 \quad \text { for } k=1 .
$$

Remark. It is clear that

$$
a_{k k-1}-a_{k k}=(k-1)\left(\begin{array}{c}
d+k \\
d
\end{array}\right) /(d+1)^{k} \text { for } c_{\left(j^{\prime}\right)}^{\prime}=c_{1}^{d+k}, 0 \leq k \leq d+1 \text {. }
$$

Theorem 4.6 in [6] and Theorem 5.1 imply the following

Corollary 5.2. The subfoliation considered in Theorem 5.1 is not integrably homotopic to a subfoliation of codimension $(d+1,2 d+1)$ on $M$ of the form $\left(F_{1}^{\prime}, F_{1}^{\prime} \cap F_{0}^{\prime}\right)$ with $F_{0}^{\prime}$ a d-codimensional foliation on $M$.

Let $\left(q_{1}, q_{2}\right)=(d+1,2 d+1)$ with $d \geq 1$. Consider the universal characteristic homomorphism

$$
\Delta_{*}^{\prime}: H\left(W\left(\operatorname{gl}\left(q_{1}\right) \oplus \operatorname{gl}(d), \mathrm{SO}\left(q_{1}\right) \times \mathrm{SO}(d)\right)_{I}\right) \rightarrow H\left(B \Gamma^{+}, R\right)
$$

(resp. $\left.\Delta_{i *}^{\prime}: H\left(W\left(\mathrm{gl}\left(q_{i}\right), \mathrm{SO}\left(q_{i}\right)\right)_{q_{i}}\right) \rightarrow H\left(B \Gamma_{q_{i}}^{+}, R\right)\right)$ for subfoliations of codimension $\left(q_{1}, q_{2}\right)$ (resp. for foliations of codimension $q_{i}, i=1,2$ ) with oriented normal bundle, and the canonical homomorphisms $\phi^{\prime *}: H\left(B \Gamma^{+}, R\right) \rightarrow$ $H\left(B \Gamma_{q_{1}}^{+} \times B \Gamma_{d}^{+}, R\right)$ and $\phi_{i}^{\prime *}: H\left(B \Gamma_{q_{i}}^{+}, R\right) \rightarrow H\left(B \Gamma^{+}, R\right), i=1,2$. Then, by Theorem 6.1 in [7], Theorem 5.1, Propositions 3.4 and 4.2, and Corollary 4.3 (in the oriented case) we obtain the following result.

Theorem 5.3. Let $z_{\left(i, j, j^{\prime}\right)}$ be cocycles as in Theorem 5.1 with $1<k \leq d+1$. Then the universal secondary characteristic classes

$$
\Delta_{*}^{\prime}\left[z_{\left(i, j, j^{\prime}\right)} \otimes \Phi\right] \in \Delta_{*}^{\prime} \operatorname{Ker} p_{*}^{\prime} \subset \operatorname{Ker} \phi^{\prime *} \subset H\left(B \Gamma^{+}, R\right)
$$

for all $2 \leq i_{1}<\cdots<i_{t_{s}}<i_{t_{s}+1}<\cdots<i_{s} \leq n, 0 \leq s \leq n-1, \Phi=1$ if $d=2 n-1$ and $\Phi=1$ or $e_{n}^{\prime}$ if $d=2 n$, are linearly independent. The corresponding classes then span a subspace $E \subset \Delta_{*}^{\prime} \operatorname{Ker} p_{*}^{\prime}$ of dimension $2^{[d / 2]}$ satisfying $E \cap \operatorname{Im} \phi_{i}^{\prime *} \Delta_{i *}^{\prime}=0, i=1,2$.

Corollary 5.4. $\operatorname{Ker} \phi^{\prime *} \neq 0$. Therefore, the canonical homomorphism $\phi_{1}^{\prime *}$ is not surjective.

Let $A \subset H\left(B \Gamma^{+}, R\right)$ be the subalgebra generated by all elements of $\operatorname{Im} \phi_{1}^{\prime *} \Delta_{1 *}^{\prime}$ $\cup \operatorname{Im} \phi_{2}^{\prime *} \Delta_{2 *}^{\prime}$. Consider the subspace $E^{\prime} \subset E$ of dimension $2^{n-1}$ spanned by the universal secondary characteristic classes $\Delta_{*}^{\prime}\left[z_{\left(i, j, j^{\prime}\right)} \otimes \Phi\right]$ given in Theorem 5.3 with $\Phi=1$ for $d=2 n-1$ and $\Phi=e_{n}^{\prime}$ for $d=2 n$. Then we have the following corollary.

Corollary 5.5. $E^{\prime} \cap A=0$.

Similarly, applying Theorem 6.1 in [7], Theorem 5.1, Propositions 3.4 and 4.2, and Corollary 4.3, we obtain the following 
Corollary 5.6. There is a subspace $\widetilde{N} \subset \operatorname{Im} \Delta_{*}^{\prime}$ of dimension $2^{[d / 2]}$, spanned by universal secondary characteristic classes, such that $\tilde{N} \cap A=0$ and $\widetilde{N} \cap \operatorname{Ker} \phi^{\prime *}=$ 0 .

Example 2. Let $\bar{G}=\mathrm{SL}(d+2), G_{1}=\mathrm{SL}(d+2,1), G_{2}=\mathrm{SL}(d+2,2)$, $H=\mathrm{O}(d)$, and $\bar{\Gamma} \subset \mathrm{SL}(d+2)$ be as in Example 1 (with $d \geq 1$ ). Consider the locally homogeneous subfoliation $\left(F_{1}, F_{2}\right)=\left(F_{G_{1}}, F_{G_{2}}\right)$ of codimension $\left(q_{1}, q_{2}\right)=(d+1,2 d+1)$ on $M=\bar{\Gamma} \mid \bar{G} / H$ (whose normal bundle is not necessarily orientable). Then, by Theorem 6.2 in [7], Theorems 5.1 and 5.3, Propositions 3.4 and 4.2, and Corollary 4.3 we obtain the following result.

Theorem 5.7. Let $\left(q_{1}, q_{2}\right)=(d+1,2 d+1)$ with $d \geq 1$. Let $z_{\left(i, j, j^{\prime}\right)}$ be cocycles as in Theorem 5.3. Then the universal secondary characteristic classes

$$
\Delta_{*}\left[z_{\left(i, j, j^{\prime}\right)}\right] \in \Delta_{*} \operatorname{Ker} p_{*} \subset \operatorname{Ker} \phi^{*} \subset H(B \Gamma, R)
$$

for all $2 \leq i_{1}<\cdots<i_{t_{s}}<i_{t_{s}+1}<\cdots<i_{s} \leq n=[(d+1) / 2], 0 \leq s \leq$ $n-1$, are linearly independent. The corresponding classes then span a subspace $E \subset \Delta_{*} \operatorname{Ker} p_{*}$ of dimension $2^{n-1}$ satisfying $E \cap \operatorname{Im} \phi_{i}^{*} \Delta_{i *}=0, i=1,2$. For $d=2 n-1$, we have $E \cap A=0$, where $A \subset H(B \Gamma, R)$ denotes the subalgebra generated by all elements of $\operatorname{Im} \phi_{1}^{*} \Delta_{1 *} \cup \operatorname{Im} \phi_{2}^{*} \Delta_{2 *}$. Furthermore, for $d=2 n-1$, there is a subspace $\widetilde{N} \subset \operatorname{Im} \Delta_{*}$ of dimension $2^{n-1}$, spanned by universal secondary characteristic classes, such that $\widetilde{N} \cap A=0$ and $\widetilde{N} \cap \operatorname{Ker} \phi^{*}=$ 0 .

Corollary 5.8. $\operatorname{Ker} \phi^{*} \neq 0$. It follows that the canonical homomorphism

$$
\phi_{1}^{*}: H\left(B \Gamma_{q_{1}}, R\right) \rightarrow H(B \Gamma, R)
$$

is not surjective.

Example 3. Let $\bar{G}, G_{1}$, and $G_{2}$ be as in Example 2, $H=\{e\}$, and $\bar{\Gamma} \subset$ $\mathrm{SL}(d+2)$ a discrete uniform subgroup (with $d \geq 1$ ). Consider the locally homogeneous subfoliation $\left(F_{1}, F_{2}\right)=\left(F_{G_{1}}, F_{G_{2}}\right)$ of codimension $\left(q_{1}, q_{2}\right)=$ $(d+1,2 d+1)$ with trivialized normal bundle on $M=\bar{\Gamma} \backslash \bar{G}$. Now, let $c_{(j)}$ and $c_{\left(j^{\prime}\right)}^{\prime}$ be as in Theorem 5.1 with $1<k \leq d+1$. Let $t_{0}, t_{1}, \ldots, t_{d-1}$ be integers such that $0 \leq t_{s} \leq s$ for $s=0,1, \ldots, d-1$. Consider in $H\left(W_{I}\right)$ the cohomology classes of the cocycles

$$
z_{\left(i, j, j^{\prime}\right)}=y_{1} \wedge y_{i_{1}} \wedge \cdots \wedge y_{i_{t_{s}}} \wedge y_{1}^{\prime} \wedge y_{i_{t_{s}+1}}^{\prime} \wedge \cdots \wedge y_{i_{s}}^{\prime} \otimes c_{(j)} c_{\left(j^{\prime}\right)}^{\prime}
$$

with $2 \leq i_{1}<\cdots<i_{t_{s}}<i_{t_{s}+1}<\cdots<i_{s} \leq d, 0 \leq s \leq d-1$, where $z_{\left(i, j, j^{\prime}\right)}=y_{1} \wedge y_{1}^{\prime} \otimes c_{(j)} c_{\left(j^{\prime}\right)}^{\prime}$ for $s=0, z_{\left(i, j, j^{\prime}\right)}=y_{1} \wedge y_{i_{1}} \wedge \cdots \wedge y_{i_{s}} \wedge y_{1}^{\prime} \otimes c_{(j)} c_{\left(j^{\prime}\right)}^{\prime}$ for $s>0$ and $t_{s}=s$, and $z_{\left(i, j, j^{\prime}\right)}=y_{1} \wedge y_{1}^{\prime} \wedge y_{i_{1}}^{\prime} \wedge \cdots \wedge y_{i_{s}}^{\prime} \otimes c_{(j)} c_{\left(j^{\prime}\right)}^{\prime}$ for $s>0$ and $t_{s}=0$. Then, by a technique analogous to that used in the proof of Theorems 5.1 and 5.3 but from more elementary computations we obtain

Theorem 5.9. Let $\left(q_{1}, q_{2}\right)=(d+1,2 d+1)$ with $d \geq 1$. Then the universal secondary characteristic classes

$$
\widetilde{\Delta}_{*}\left[z_{\left(i, j, j^{\prime}\right)}\right] \in \widetilde{\Delta}_{*} \operatorname{Ker} \tilde{p}_{*} \subset \operatorname{Ker} \tilde{\phi}^{*} \subset H(F \Gamma, R)
$$

for all $2 \leq i_{1}<\cdots<i_{t_{s}}<i_{t_{s}+1}<\cdots<i_{s} \leq d, 0 \leq s \leq d-1$, are linearly independent. The corresponding classes then span a subspace $E \subset \widetilde{\Delta}_{*} \operatorname{Ker} \tilde{p}_{*}$ of dimension $2^{d-1}$ satisfying $E \cap \operatorname{Im} \tilde{\phi}_{i}^{*} \widetilde{\Delta}_{i *}=0, i=1,2$. 
Corollary 5.10. For $\left(q_{1}, q_{2}\right)=(d+1,2 d+1)$ with $d \geq 1$, the canonical homomorphism $\tilde{\phi}^{*}: H(F \Gamma, R) \rightarrow H\left(F \Gamma_{q_{1}} \times F \Gamma_{d}, R\right)$ is not injective. Hence, the canonical homomorphism $\tilde{\phi}_{1}^{*}: H\left(F \Gamma_{q_{1}}, R\right) \rightarrow H(F \Gamma, R)$ is not surjective.

Remark. Let $\left(q_{1}, q_{2}\right)$ be a couple of integers with $0<q_{1}<q_{2}$. It follows from $[11,14,15]$ that the canonical homomorphisms $\phi^{*}, \phi^{\prime *}, \tilde{\phi}^{*}, \phi_{2}^{*}, \phi_{2}^{\prime *}$ and $\tilde{\phi}_{2}^{*}$ are not surjective.

Proposition 5.11. Let $\left(q_{1}, q_{2}\right)$ be a couple of integers with $0<q_{1}<q_{2},\left(q_{1}, q_{2}\right)$ $\neq(2 m-1,2 m)$, and $\left(q_{1}, q_{2}\right) \neq(1,2 m)$. Then the canonical homomorphism $\phi_{2}^{*}: H\left(B \Gamma_{q_{2}}, R\right) \rightarrow H(B \Gamma, R)$ is not injective.

Proof. Consider in $H\left(W \mathrm{O}_{q_{2}}\right)$ the cohomology class of a monomial cocycle of the form

$$
z=y_{1}^{\prime \prime} \wedge y_{2 q_{2}^{\prime}-1}^{\prime \prime} \otimes c_{(j)}^{\prime \prime}=y_{1}^{\prime \prime} \wedge y_{2 q_{2}^{\prime}-1}^{\prime \prime} \otimes c_{1}^{\prime \prime j_{1}} \cdots c_{q_{2}}^{\prime \prime} j_{q_{2}}
$$

with $\operatorname{deg} c_{(j)}^{\prime \prime}=2 q_{2}$, where $q_{2}^{\prime}=\left[\left(q_{2}+1\right) / 2\right] \geq 2$. Then, from [7] it follows that $\Delta_{2 *}[z] \neq 0 \in H\left(B \Gamma_{q_{2}}, R\right)$. On the other hand, by virtue of Corollary 5.2 in [7], the cohomology class $[z]$ is in the kernel of the canonical homomorphism $W\left(d \rho_{2}\right)^{*}: H\left(W \mathrm{O}_{q_{2}}\right) \rightarrow H\left(W \mathrm{O}_{I}\right)$. Whence, in view of Proposition 3.4, we have $\Delta_{2 *}[z] \in \operatorname{Ker} \phi_{2}^{*}$.

Remarks. (1) It is clear that $\Delta_{2 *}\left[1 \otimes c_{q_{2}}^{\prime \prime}\right] \in \operatorname{Ker} \phi_{2}^{*}$ for $\left(q_{1}, q_{2}\right)=(2 n-1,2 m)$ with $0<q_{1}<q_{2}$. Unfortunately, we have been unable to prove that $\Delta_{2 *}\left[1 \otimes c_{q_{2}}^{\prime \prime}\right] \neq 0$.

(2) A geometric interpretation for nontrivial elements of the kernel of the canonical homomorphism $W\left(d \rho_{2}\right)^{*}$ has been given in [7] (see also [5]).

(3) In a similar way, we show that the canonical homomorphisms

$$
\phi_{2}^{\prime *}: H\left(B \Gamma_{q_{2}}^{+}, R\right) \rightarrow H\left(B \Gamma^{+}, R\right)
$$

and

$$
\tilde{\phi}_{2}^{*}: H\left(F \Gamma_{q_{2}}, R\right) \rightarrow H(F \Gamma, R)
$$

are not injective for $0<q_{1}<q_{2}$ Evidently, $\Delta_{*}^{\prime}\left[1 \otimes e_{m}^{\prime \prime}\right] \in \operatorname{Ker} \phi_{2}^{\prime *}$ is not zero for $\left(q_{1}, q_{2}\right)=(2 n-1,2 m)$ with $0<q_{1}<q_{2}$, where $e_{m}^{\prime \prime} \in I^{2 m}\left(\operatorname{SO}\left(q_{2}\right)\right)$ is the Pfaffian polynomial for $q_{2}=2 \mathrm{~m}$. Analogously, it is easily shown that the element $\widetilde{\Delta}_{*}\left[y_{1}^{\prime \prime} \wedge y_{q_{2}}^{\prime \prime} \otimes c_{(j)}^{\prime \prime}\right] \in \operatorname{Ker} \tilde{\phi}_{2}^{*}$ is not zero for $\operatorname{deg} c_{(j)}^{\prime \prime}=2 q_{2}$ with $0<q_{1}<q_{2}$.

\section{REFERENCES}

1. C. Benson and D. B. Ellis, Characteristic classes of transversely homogeneous foliations, Trans. Amer. Math. Soc. 289 (1985), 849-859.

2. R. Bott, Lectures on characteristic classes and foliations, Lecture Notes in Math., vol. 279, Springer-Verlag, Berlin and New York, 1972, pp. 1-94.

3. J. M. Carballés, Characteristic homomorphism for $\left(F_{1}, F_{2}\right)$-foliated bundles over subfoliated manifolds, Ann. Inst. Fourier (Grenoble) 33 (1984), 219-245.

4. L. A. Cordero, Sheaves and cohomologies associated to subfoliations, Results in Math. 8 (1985), 9-20.

5. L. A. Cordero and X. Masa, Characteristic classes of subfoliations, Ann. Inst. Fourier (Grenoble) 31 (1981), 61-86.

6. D. Domínguez, Sur les classes caractéristiques des sous-feuilletages, Publ. Res. Inst. Math. Sci. 23 (1987), 813-840. 
7. __ Classes caractéristiques non triviales de sous-feuilletages localement homogènes, Kodai Math. J. 11 (1988), 177-204.

8. __ Classes caractéristiques non triviales des feuilles de sous-feuilletages localement homogènes, Geom. Dedicata 28 (1988), 229-249.

9. B. L. Feigin, Characteristic classes of flags of foliations, Functional Anal. Appl. 9 (1975), 312-317.

10. D. B. Fuks, Non-trivialité des classes caractéristiques de g-structures. Applications aux classes caractéristiques de feuilletages, C. R. Acad. Sci. Paris 284 (1977), 1017-1019.

11. __ Non-trivialité des classes caractéristiques de g-structures. Applications aux variations des classes caractéristiques de feuilletages, C. R. Acad. Sci. Paris 284 (1977), 1105-1107.

12. A. Haefliger, Homotopy and integrability, Lecture Notes in Math., vol. 197, Springer-Verlag, Berlin and New York, 1971, pp. 133-163.

13. __ Sur les classes caractéristiques des feuilletages, Lecture Notes in Math., vol. 317, Springer-Verlag, Berlin and New York, 1973, pp. 239-260.

14. J. L. Heitsch, Deformations of secondary characteristic classes, Topology 12 (1973), 381-388.

15. _ Independent variation of secondary classes, Ann. of Math. (2) 108 (1978), 421-460.

16. F. W. Kamber and Ph. Tondeur, Foliated bundles and characteristic classes, Lecture Notes in Math., vol. 493, Springer-Verlag, Berlin and New York, 1975.

17. Stud., vol. 5, Academic Press, New York and London, 1979, pp. 213-263.

18. R. Moussu, Sur les classes exotiques des feuilletages, Lecture Notes in Math., vol. 392, Springer-Verlag, Berlin and New York, 1974, pp. 37-42.

19. R. A. Wolak, Characteristic classes of almost-flag structures, Geom. Dedicata 24 (1987), 207-220.

Departamento de Matemáticas, Estadistica y Computación, facultad de Ciencias, UNIVERSIDAD DE CANTABRIA, 39071 SANTANDER, SPAIN 